\title{
Article \\ Deposition of Crystalline GdIG Samples Using Metal Organic Decomposition Method
}

\author{
Hyeongyu Kim ${ }^{1,+}{ }^{+}$Phuoc-Cao Van ${ }^{2,+}{ }^{-}$, Hyeonjung Jung ${ }^{3,+}{ }^{,}$Jiseok Yang ${ }^{1}$, Younghun Jo ${ }^{4}$, Jung-Woo Yoo ${ }^{3}$, \\ Albert M. Park ${ }^{1, *}$, Jong-Ryul Jeong ${ }^{2, *(D)}$ and Kab-Jin Kim ${ }^{1, *}$ \\ 1 Department of Physics, Korean Advanced Institute of Science and Technology, Daejeon 34141, Korea; \\ kimkkol@kaist.ac.kr (H.K.); jiseok@kaist.ac.kr (J.Y.) \\ 2 Department of Materials Science and Engineering, Chungnam National University, Daejeon 34148, Korea; \\ caovanphuoc91@gmail.com \\ 3 Department of Materials Science and Engineering, Ulsan National Institute of Science and Technology, \\ Ulsan 44919, Korea; $111111151 @$ unist.ac.kr (H.J.); jwyoo@unist.ac.kr (J.-W.Y.) \\ 4 Division of Scientific Instrumentation, Korean Basic Science Institute, Daejeon 34126, Korea; \\ younghun@kbsi.re.kr \\ * Correspondence: bertpark@kaist.ac.kr (A.M.P.); jrjeong@cnu.ac.kr (J.-R.J.); kabjin@kaist.ac.kr (K.-J.K.) \\ + These authors contributed equally to this work.
}

check for

updates

Citation: Kim, H.; Van, P.-C.; Jung,

H.; Yang, J.; Jo, Y.; Yoo, J.-W.; Park,

A.M.; Jeong, J.-R.; Kim, K.-J.

Deposition of Crystalline GdIG

Samples Using Metal Organic

Decomposition Method.

Magnetochemistry 2022, 8, 28.

https://doi.org/10.3390/

magnetochemistry 8030028

Academic Editor: Atsufumi Hirohata

Received: 31 December 2021

Accepted: 22 February 2022

Published: 27 February 2022

Publisher's Note: MDPI stays neutral with regard to jurisdictional claims in published maps and institutional affiliations.

Copyright: (C) 2022 by the authors. Licensee MDPI, Basel, Switzerland. This article is an open access article distributed under the terms and conditions of the Creative Commons Attribution (CC BY) license (https:/ / creativecommons.org/licenses/by/ $4.0 /)$.

\begin{abstract}
Fabrication of high quality ferrimagnetic insulators is an essential step for ultrafast magnonics, which utilizes antiferromagnetic exchange of the ferrimagnetic materials. In this work, we deposit high-quality GdIG thin films on a (111)-oriented GGG substrate using the Metal Organic Decomposition (MOD) method, a simple and high throughput method for depositing thin film materials. We postannealed samples at various temperatures and examined the effect on structural properties such as crystallinity and surface morphology. We found a transition in the growth mode that radically changes the morphology of the film as a function of annealing temperature and obtained an optimal annealing temperature for a uniform thin film with high crystallinity. Optimized GdIG has a high potential for spin wave applications with a low damping parameter in the order of $10^{-3}$, which persists down to cryogenic temperatures.
\end{abstract}

Keywords: garnet ferrite; compensated ferrimagnet; metal organic decomposition

\section{Introduction}

High frequency dynamics from the antiferromagnetic exchange are essential for achieving fast computing spintronic devices [1,2]. On the other hand, to utilize the antiferromagnetic dynamics, a method to overcome its lack of responsiveness to the external field needs to be devised. Compensated ferrimagnet, composed of two antiferromagnetically coupled sublattices [3], enables the fast dynamics of antiferromagnetic materials while having accessibility via external fields similar to ferromagnetic ones.

The spin wave carries information as a collective precessional motion of localized magnetization. Unlike electric current mediated by the motion of charge carriers, the spin wave does not involve joule heating when propagating. Thus, it has high potential in the applications such as low-power computing devices [3]. However, a low damping constant is required to ensure a long transport distance for practical application. Rare earth iron garnet (REIG) is one of the best candidates in ferrimagnetic magnon transport. As garnet ferrite is an insulator, there is no electron spin sink contribution to damping, which results in low damping [4].

One of the obstacles in using REIG is a complicated fabrication process [5], which requires a dedicated vacuum chamber [6] or annealing temperature near the melting point [6-8]. The metal organic decomposition (MOD) method provides a simple and reproducible method to deposit REIG. Unlike other methods, the MOD method only needs 
a spin coating of the inorganic compound solution and the annealing temperature lower than the melting point.

In this work, we prepared gadolinium iron garnet (GdIG) thin film on gadolinium gallium garnet (GGG) substrate using the MOD method. X-ray diffraction (XRD), transmission electron microscopy (TEM), superconducting quantum interference device vibrational sample magnetometer (SQUID VSM), and Atomic force microscope (AFM) were applied to check crystallinity, magnetic properties, and morphology of samples. We found that high-quality garnet samples with reproducible magnetic properties can be deposited, which can be used to study physical phenomena such as longitudinal spin Seebeck effect [9] and spin Hall effect-driven various magnetoresistances [10,11].

\section{Materials and Methods}

The precursor solution is prepared by dissolving inorganic compounds $\mathrm{Gd}\left(\mathrm{NO}_{3}\right)_{3} \cdot 6 \mathrm{H}_{2} \mathrm{O}$ with $99.99 \%$ purity (from Alfa Aesar, Ward Hill, MA, USA) and $\mathrm{Fe}\left(\mathrm{NO}_{3}\right)_{3} \cdot 6 \mathrm{H}_{2} \mathrm{O}$, which has $99.9 \%$ purity (from Sigma Aldrich, St. Louis, MO, USA), into the solvent. A mixture of $99.8 \%$ purity dimethylformamide (DMF/Sigma Aldrich, St. Louis, MO, USA) and 95\% purity polyvinylpyrrolidone (PVP) was applied as a solvent [12]. DMF and PVP were chosen because the mixture can dissolve inorganic compounds and, hence, can be utilized to grow garnet samples using MOD method [12-14]. As the mixing rate of DMF and PVP can dominantly affect the thickness and morphology of the sample, we used the reported values for YIG and BiYIG [13] because they have similar structures with GdIG. The stoichiometry of the Fe compound and Gd compound was set to 3:5, based on the formula unit of $\mathrm{Gd}_{3} \mathrm{Fe}_{5} \mathrm{O}_{12}$. The total concentration of metallic compounds was fixed to $17 \%$.

In order to avoid the contamination at surface of substrate, the GGG (111) substrate was sonicated first in acetone and then in ethanol for $30 \mathrm{~min}$ each. After that, the sample was treated under Ar plasma ( $20 \mathrm{sccm}$ of Ar, $100 @$ power with $70 \mathrm{kHz}$ ) for $30 \mathrm{~min}$. A MOD solution was spin-coated onto GGG (111) substrate at $500 \mathrm{rpm}$ for $5 \mathrm{~s}$ and $3000 \mathrm{rpm}$ for $30 \mathrm{~s}$. The coating and solution preparing procedure was optimized to deposit $10 \mathrm{~nm}$ of garnet sample [12]. For thicker samples, we repeated the growth procedure multiple times. After coating, the sample was dried for $30 \mathrm{~min}$ at $100^{\circ} \mathrm{C}$ using a hot plate. The sample was then annealed for $1 \mathrm{~h}$ under an oxygen-rich atmosphere at various temperatures $\left(750^{\circ} \mathrm{C}, 800^{\circ} \mathrm{C}\right.$, $900{ }^{\circ} \mathrm{C}$, and $1000{ }^{\circ} \mathrm{C}$ ) to determine the optimal annealing temperature for high crystallinity and good surface morphology. A fixed ramping rate of $4{ }^{\circ} \mathrm{C} / \mathrm{min}$ was used to reach the target temperature.

Crystallinity was confirmed using a high-resolution XRD (RIGAKU corporation) with $\mathrm{K}_{\alpha}$ emission line of Copper $(\lambda=1.54 \AA$ ). Surface morphology was examined by using AFM (XE-7 designed by Park Systems). Magnetic property, especially the magnetization compensation temperature $\left(T_{M}\right)$, was measured by SQUID VSM in the Quantum Design magnetic property measurement system (MPMS).

\section{Results}

\subsection{Structural Properties}

To find the optimal post-annealing temperature for crystal formation, we first deposited a GdIG sample, targeting $10 \mathrm{~nm}$ thickness on the GGG substrate. Figure 1a,b show the measured XRD spectrum of deposited GdIG samples for various annealing temperatures. GGG (444) peak appears at $51.2^{\circ}$, which indicates that the lattice constant of GGG is $12.37 \AA$. Next to GGG (444) peak, a broad peak is observed at $50.3^{\circ}$, which corresponds to the GdIG (444) peak with a lattice constant of $12.55 \AA$. We note that the lattice constant of GdIG on GGG is larger than that of a single crystalline GdIG of $12.48 \AA$ due to the pseudo-morphic growth on the GGG substrate [15]. We confirmed that there is no extra peak in the entire XRD pattern (Figure 1a) except for 111 orientation, suggesting the high epitaxial quality of GdIG. The thickness of GdIG was confirmed using a fringe pattern in the XRD spectrum. For the $750{ }^{\circ} \mathrm{C}$ annealed sample, the first and second fringe peaks 
were observed at $52.08^{\circ}$ and $52.98^{\circ}$, from which the thickness of GdIG was estimated to be $11 \pm 2 \mathrm{~nm}$. This confirms that the thickness of GdIG is almost the same as what we targeted.

(a)

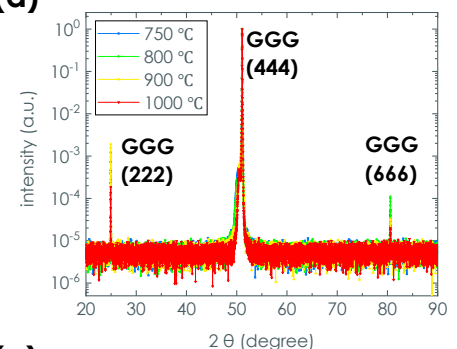

(c)

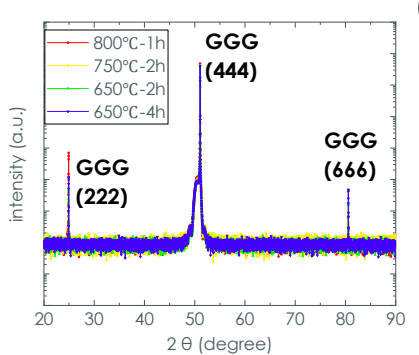

(b)

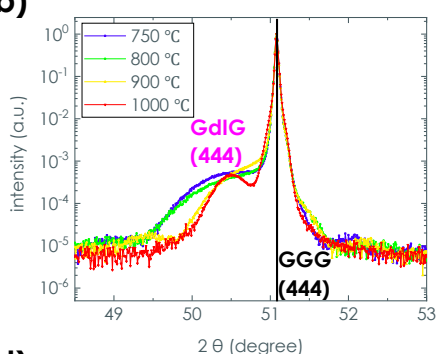

(d)

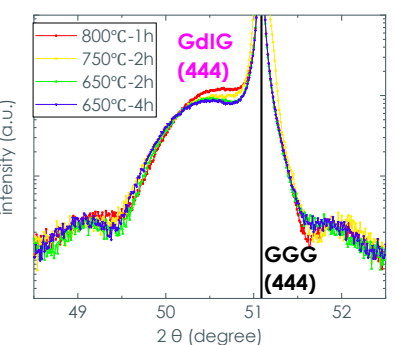

Figure 1. XRD spectrum for GdIG samples annealed at various annealing temperatures (a) from $20^{\circ}$ to $90^{\circ}$ and (b) from $48.5^{\circ}$ to $53^{\circ}$. XRD spectrum for GdIG samples annealed at various annealing times (c) from $20^{\circ}$ to $90^{\circ}$ and (d) from $48.5^{\circ}$ to $53^{\circ}$.

As shown in Figure 1b, the increased annealing temperature drives GdIG peak shaper. As the broadening of XRD peak is caused by vacancy- or defect-driven lattice expansion, the $X R D$ result suggests that the higher annealing temperature results in better crystallinity [5]. We also confirmed that annealing time does not exert any significant change in the XRD pattern (Figure 1c). This suggests that crystallinity is predominantly governed by the annealing temperature.

However, we find that the high annealing temperature can affect the surface topography. The surface topography map measured using AFM in the non-contact mode (Figure 2) shows a significant change in surface morphology as annealing temperature increases. The roughness of film surface is about $0.1 \mathrm{~nm}$ order for the $750^{\circ} \mathrm{C}$ and $800{ }^{\circ} \mathrm{C}$ annealed samples (see Table 1). However, the surface morphology of GdIG changes abruptly above $900^{\circ} \mathrm{C}$, and the island starts to appear. For $1000{ }^{\circ} \mathrm{C}$ annealed samples, island growth becomes the dominant film formation mechanism, resulting in the entire area being covered with islands of $\sim 200 \mathrm{~nm}$ diameter and $\sim 20 \mathrm{~nm}$ height.

Table 1. Roughness of films (Ra) for several annealing temperatures.

\begin{tabular}{cc}
\hline Annealing Temperature $\left({ }^{\circ} \mathbf{C}\right)$ & Ra (nm) \\
\hline 750 & 0.14 \\
800 & 0.08 \\
900 & 0.71 \\
1000 & 8.71 \\
\hline
\end{tabular}

As the purpose of this work is to deposit uniform film for spin wave application, we selected $7500^{\circ} \mathrm{C}$ postannealed sample and performed additional measurement using TEM to ensure the crystallinity. TEM measurement was conducted using the STEM (scanning transmission electron microscope) system with Cs-corrector (JEM-2100F) designed by JEOL. A high-resolution TEM image in Figure 3a shows that GdIG and GGG have a smooth interface with a continuous crystal structure above and below the interface due to a similar lattice constant between the two materials. Additionally, a clear separation of Fe in the 
GdIG film and Ga in the GGG substrate from energy dispersive X-ray spectroscopy (EDX) (Figure $3 b$ ) indicates minimal mixing between GdIG and substrate.
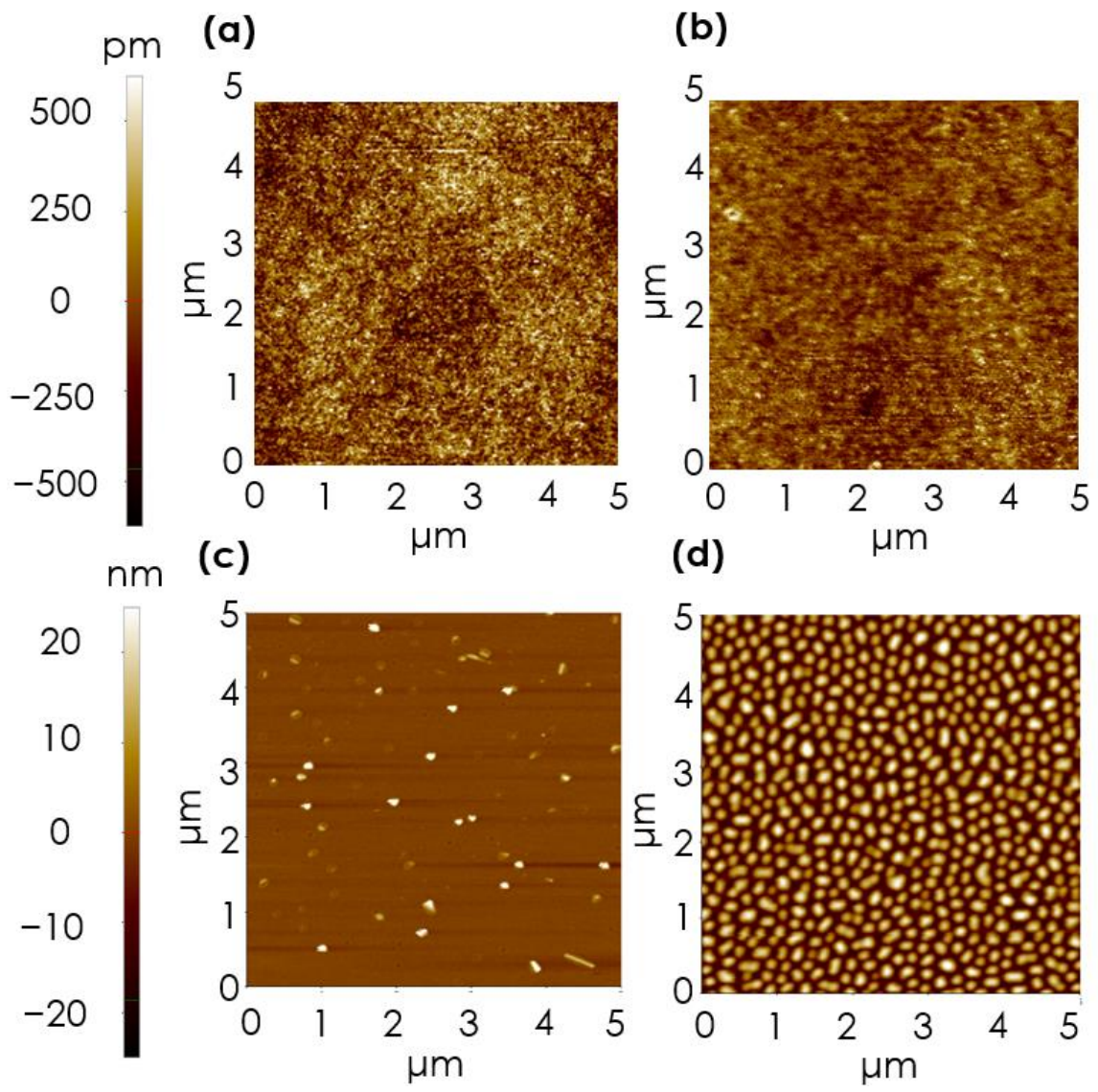

Figure 2. AFM image of (a) $750{ }^{\circ} \mathrm{C},(\mathbf{b}) 800{ }^{\circ} \mathrm{C}$, (c) $900{ }^{\circ} \mathrm{C}$, and (d) $1000{ }^{\circ} \mathrm{C}$ annealed samples.

(a)

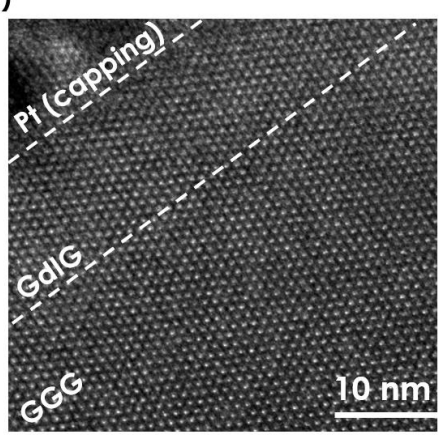

(b)

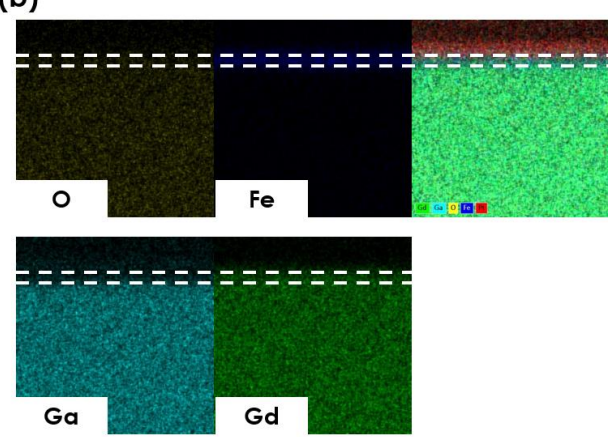

Figure 3. (a) TEM and (b) EDX images of $750^{\circ} \mathrm{C}$ annealed GGG I GdIG sample. As GGG and GdIG have similar structure and lattice parameters, a smooth interface appears, indicating an epitaxial growth of GdIG. (b) The presence of Gd and Fe and the absence of $\mathrm{Ga}$ at the marked region represent the formation of the GdIG layer.

\subsection{Magnetic Property}

The magnetic property was checked as an additional measure to ensure crystallinity and stoichiometry. Temperature dependent magnetometry of $10 \mathrm{~nm}$ GdIG sample annealed at $750{ }^{\circ} \mathrm{C}$ was performed using SQUID VSM in MPMS 3. An M-T curve was obtained by measuring $\mathrm{M}-\mathrm{H}$ curves under in-plane magnetic field at various temperatures and extracting the saturation magnetization $\left(M_{\mathrm{S}}\right)$. We eliminated paramagnetic contribution from GGG substrate by subtracting the linear background in the M-H curve at each temperature. 
Figure 4 shows the extracted M-T curve. We focus on the value of $M_{S}$ at low temperatures and the magnetic compensation point. $M_{S}$ at $20 \mathrm{~K}$ was about $700 \mathrm{emu} / \mathrm{cc}$, similar to previous reports as shown in Table 2. Magnetic compensation point appeared near $270 \mathrm{~K}$ also agrees with references on bulk and thin film GdIG $[9,16,17]$.

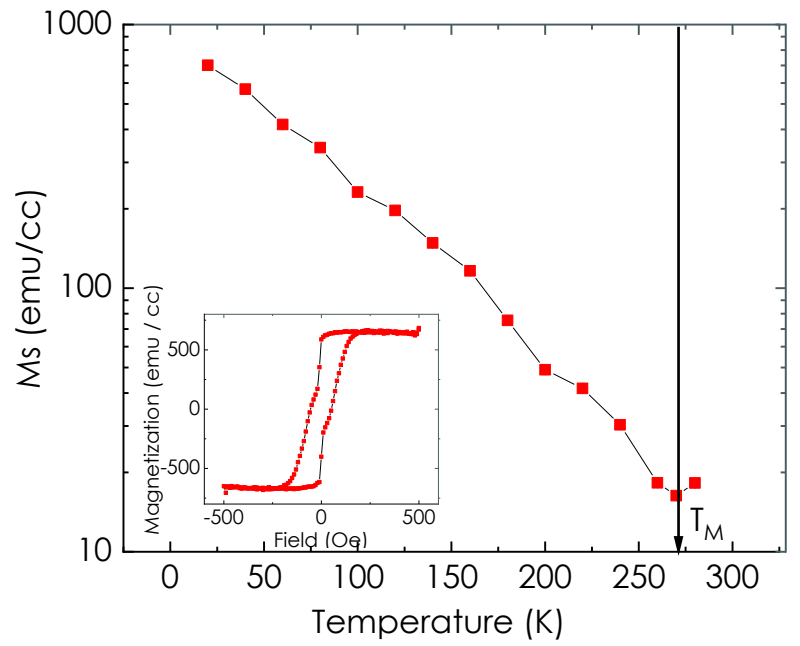

Figure 4. M-T curve of GdIG $10 \mathrm{~nm}$ thin film post-annealed at $750{ }^{\circ} \mathrm{C}$. Inset shows the $\mathrm{M}-\mathrm{H}$ curve at $20 \mathrm{~K}$.

Table 2. Compensation temperature $T_{\mathrm{M}}$ and saturation magnetization (Ms) at $20 \mathrm{~K}$ of GdIG.

\begin{tabular}{ccc}
\hline Source & $\boldsymbol{T}_{\mathbf{M}}$ & Ms $(\mathbf{e m u} / \mathbf{c c})$ \\
\hline From Figure 4 & $270 \mathrm{~K}$ & 700 \\
From [16] & $280 \mathrm{~K}$ & 600 \\
From [17] & $300 \mathrm{~K}$ & 670 \\
From [9] & $280 \mathrm{~K}$ & 1300 \\
\hline
\end{tabular}

\subsection{FMR Measurement}

Finally, the damping parameter of the GdIG sample was measured using the temperature dependent FMR. We used 60-nanometer-thick GdIG sample to enhance RF absorption power. Temperature and field control was performed using a physical property measurement system (PPMS). RF field for FMR was applied using a custom-built FMR setup that is compatible with PPMS.

Figure 5a shows the FMR spectra of the GdIG film for various temperatures. Here, we fixed the excitation frequency $(15 \mathrm{GHz})$ and swept the magnetic field from 0 to $5000 \mathrm{Oe}$ along the in-plane direction. Figure $5 b$ shows resonance frequency as a function of external magnetic field for $\mathrm{T}=50 \mathrm{~K}$. The exact overlap with Kittel formula (red solid line) was observed. We extracted the damping parameter from the spectrum by measuring peak to peak distance from the FMR lineshape in Figure 5a. We first use the model for conventional ferromagnetic material to obtain damping parameter $\alpha_{F M}$ :

$$
\Delta H=\Delta H_{0}+\frac{4 \pi \alpha_{F M}}{\sqrt{3} \gamma_{e f f}} f
$$

where $\gamma_{e f f}=g_{e f f} \mu_{B} / \hbar=\Delta M / \Delta S$ is the effective gyromagnetic ratio of the net moment, and $\Delta M$ and $\Delta S$ are the differences in the net moment and the spin density of two sublattices, respectively. Figure $5 c$ shows $\Delta H$ as a function of frequency for several temperaures. The slope increases with increasing temperaure. Considering that the temperature variation of $\gamma_{e f f}$ is small, as seen in Table 3, the result suggests that $\alpha_{F M}$ increased with increasing temperature, which we ascribed to the divergence of $\alpha_{F M}$ at angular momentum compensation point $\left(\mathrm{T}_{\mathrm{A}}\right)[18-20]$. 
(a)

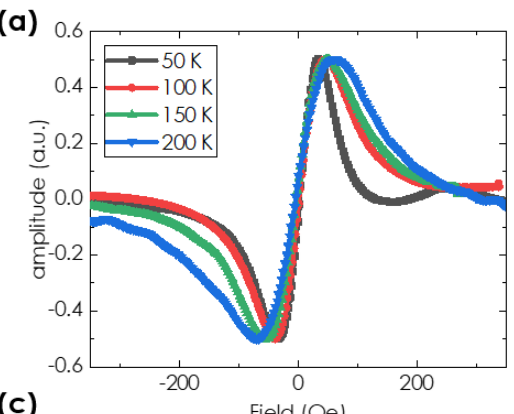

(c)

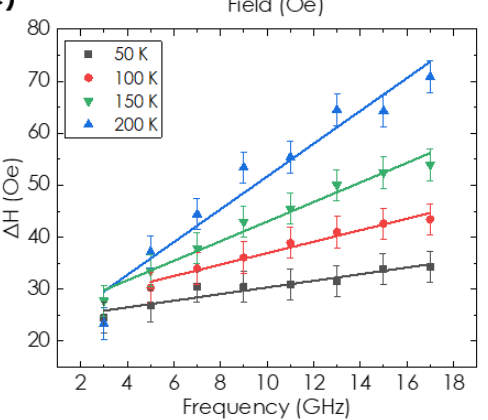

(b)

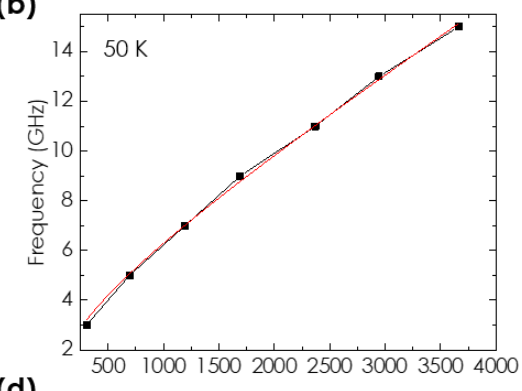

(d)

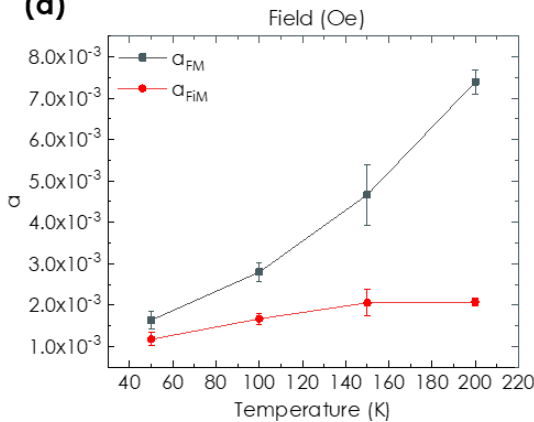

Figure 5. (a) FMR spectra of GdIG $60 \mathrm{~nm}$ samples at $15 \mathrm{GHz}$ RF frequency. The horizonal axis (field axis) is shifted as much as the resonance field to compare the linewidth for different temperatures. (b) Resonance frequency as a function of the external field at $50 \mathrm{~K}$ and the Kittel formula fitting (red line). (c) Frequency dependence of peak-to-peak FMR linewidth. The solid line represents linear fitting. (d) Temperature dependence of damping parameters $\alpha_{F M}$ and $\alpha_{F i M}$.

Table 3. Effective gyromagnetic ratio $\left(\gamma_{e f f}\right)$ and damping parameter $\left(\alpha_{F M}\right)$ measured at various temperatures below $T_{\mathrm{M}}$.

\begin{tabular}{ccc}
\hline Temperature (K) & $\gamma_{\text {eff }}\left(\times \mathbf{1 0}^{\mathbf{7}} \mathbf{T}^{-\mathbf{1}} \mathbf{s}^{-\mathbf{1}}\right)$ & $\alpha_{F M}\left(\times \mathbf{1 0}^{-\mathbf{3}}\right)$ \\
\hline 50 & $1.78 \pm 0.08$ & $1.60 \pm 0.22$ \\
100 & $1.85 \pm 0.07$ & $2.80 \pm 0.22$ \\
150 & $1.81 \pm 0.02$ & $4.67 \pm 0.74$ \\
200 & $1.75 \pm 0.08$ & $7.41 \pm 0.3$ \\
\hline
\end{tabular}

In the case of ferrimagnet, however, the dissipation rate from the two lattices and the dependence of resonance frequency on net spin density need to be considered to deduce a well-defined damping constant [18-21]. Since the GdIG sample also can be regarded as a compensated ferrimagnet with two effective sublattices, damping $\alpha_{F i M}$ is expressed as follows:

$$
\alpha_{F i M}=\frac{\left|s_{\text {net }}\right|}{\left|s_{\text {total }}\right|} \alpha_{F M}
$$

where $s_{n e t}=s_{1}-s_{2}$ and $s_{\text {total }}=s_{1}+s_{2}$ are the difference and the sum of the spin density for each sublattice $\left(s_{1,2}\right)$, respectively.

To estimate $\alpha_{F i M}$, we should extract the spin density of each sublattice in GdIG. This was achieved by using the M-T curve shown in Figure 4 and by assuming that the spin density of the Fe sublattice follows the same trend with that in YIG [16]. As shown in Figure $5 \mathrm{~d}, \alpha_{F i M}$ had no singular behavior close to angular momentum compensation in contrast to $\alpha_{F M}$, and this dependence corresponds to previous reports on $\alpha_{F i M}$ of compensated ferrimagnets, including metallic GdFeCo [20].

We note that the GdIG sample deposited by the MOD method has comparable damping constant to some of the literature on spin wave applications [22,23]. In the case of the $10 \mathrm{~nm}$ YIG sample grown by the Pulsed Laser Deposition (PLD) method, an order of $10^{-3}$ damping parameter was obtained, which is similar to our result [24]. Our data also 
suggest that the MOD-deposited GdIG can be an alternative platform that could be used for low-temperature magnonic applications.

\section{Conclusions}

A high-quality GdIG thin film on GGG substrate was prepared using the MOD method. We found that a transition of the growing model of GdIG occurs at around $900{ }^{\circ} \mathrm{C}$ above which the film grows with an island pattern. The optimal postannealing temperature was decided based on the growth mode of the film and the crystallinity confirmed by the TEM. Temperature dependence of magnetization was measured using SQUID VSM and confirmed that $T_{\mathrm{M}}$ was measured at $270 \mathrm{~K}$. FMR measurement showed that MOD deposited GdIG exhibits comparable damping constant to various deposition methods. Moreover, our experiment found that the damping constant of GdIG decreased at lower temperatures. We expect that the MOD method provides a simple and high-throughput procedure to deposit GdIG, which has potential advantages for magnonic applications.

Author Contributions: K.-J.K. and J.-R.J. planned and supervised the study. P.-C.V. deposited the GdIG sample; H.J. and J.-W.Y. performed the FMR measurement. J.Y. measured AFM; Y.J. measured MPMS; H.K. and A.M.P. analyzed the data from AFM, XRD, FMR, TEM, EDX, and MPMS and wrote the manuscript. All authors were involved in the discussion of the results and commented on the manuscript. All authors have read and agreed to the published version of the manuscript.

Funding: This research was supported by the National Research Foundation of Korea (NRF) funded by the Korean Government (MSIP) (grant numbers: 2016R1A5A1008184, and 2020R1A2C100613612) and by KAIST; funded Global Singularity Research Program for 2021; and by Samsung Research Funding \& Incubation Center of Samsung Electronics under Project Number SRFC-MA2002-02. Y.J. acknowledges the support by KBSI grant (No. D210200).

Institutional Review Board Statement: Not applicable.

Informed Consent Statement: Not applicable.

Data Availability Statement: Derived data supporting the findings of this study are available from the corresponding author (A.M.P., J.-R.J. and K.-J.K.) on request.

Conflicts of Interest: The authors declare no conflict of interest.

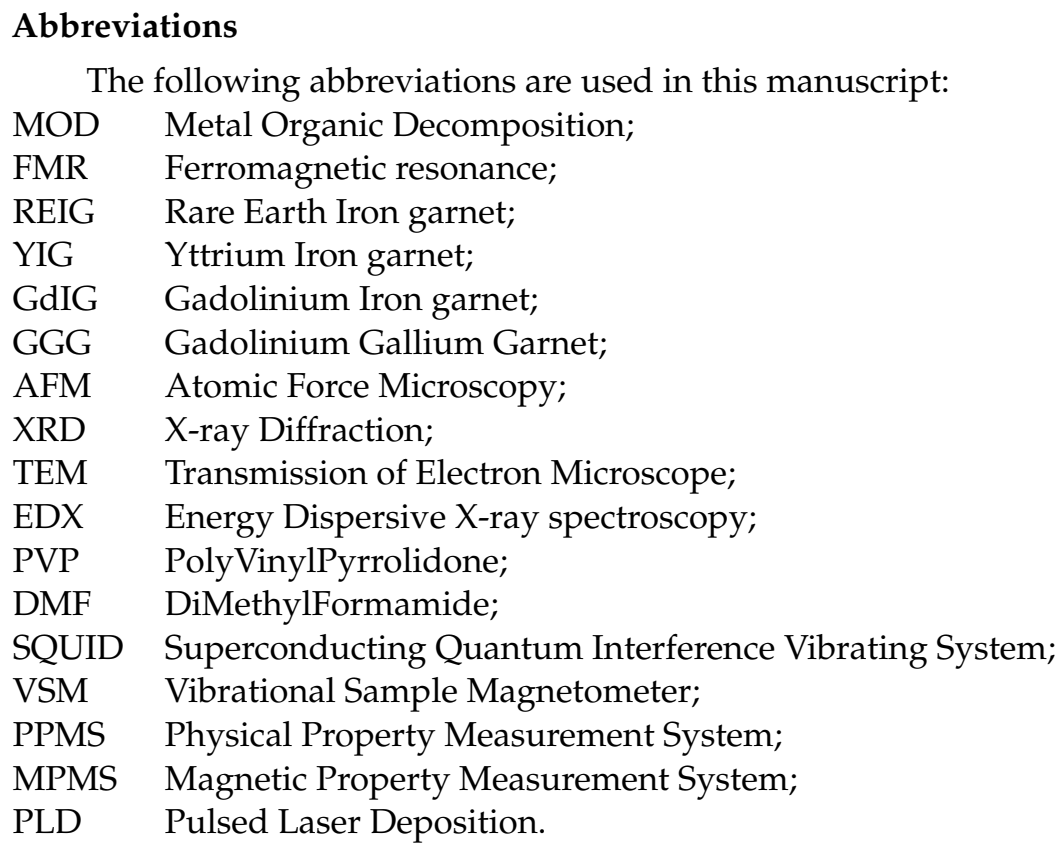




\section{References}

1. Keffer, F.; Kittel, C. Theory of antiferromagnetic resonance. Phys. Rev. 1952, 85, 329. [CrossRef]

2. Keffer, F.; Kaplan, H.; Yafet, Y. Spin Waves in Ferromagnetic and Antiferromagnetic Materials. Am. J. Phys. 1953, $21,253$. [CrossRef]

3. Kim, C.; Lee, S.; Kim, H.G.; Park, J.H.; Moon, K.W.; Park, J.Y.; Yuk, J.M.; Lee, K.J.; Park, B.G.; Kim, S.K.; et al. Distinct handedness of spin wave across the compensation temperatures of ferrimagnets. Nat. Mater. 2020, 19, 980-985. [CrossRef] [PubMed]

4. Zhang, S.; Li, Z. Roles of nonequilibrium conduction electrons on the magnetization dynamics of ferromagnets. Phys. Rev. Lett. 2004, 93, 127204. [CrossRef]

5. Cao Van, P.; Surabhi, S.; Dongquoc, V.; Kuchi, R.; Yoon, S.G.; Jeong, J.R. Effect of annealing temperature on surface morphology and ultralow ferromagnetic resonance linewidth of yttrium iron garnet thin film grown by rf sputtering. Appl. Surf. Sci. 2018, 435, 377-383. [CrossRef]

6. Gomi, M.; Tanida, T.; Abe, M. Rf sputtering of highly Bi-substituted garnet films on glass substrates for magneto-optic memory. J. Appl. Phys. 1985, 57, 3888. [CrossRef]

7. Lee, H.; Yoon, Y.; Kim, S.; Yoo, H.K.; Melikyan, H.; Danielyan, E.; Babajanyan, A.; Ishibashi, T.; Friedman, B.; Lee, K. Preparation of bismuth substituted yttrium iron garnet powder and thin film by the metal-organic decomposition method. J. Cryst. Growth 2011, 329, 27-32. [CrossRef]

8. Uchida, T.; Watanabe, S.; Uchiyama, T.; Tachiki, T. Fabrication of STO buffer films on MgO substrates by the MOD method. $J$ Phys. Conf. Ser. 2008, 97, 012057. [CrossRef]

9. Geprägs, S.; Kehlberger, A.; Coletta, F.D.; Qiu, Z.; Guo, E.J.; Schulz, T.; Mix, C.; Meyer, S.; Kamra, A.; Althammer, M.; et al. Origin of the spin Seebeck effect in compensated ferrimagnets. Nat. Commun. 2016, 7, 10452. [CrossRef]

10. Liu, G.; Wang, X.; Luan, Z.Z.; Zhou, L.F.; Xia, S.Y.; Yang, B.; Tian, Y.Z.; Guo, G.; Du, J.; Wu, D. Magnonic Unidirectional Spin Hall Magnetoresistance in a Heavy-Metal-Ferromagnetic-Insulator Bilayer. Phys. Rev. Lett. 2021, 127, 207206. [CrossRef]

11. Dong, B.W.; Cramer, J.; Ganzhorn, K.; Yuan, H.Y.; Guo, E.J.; Goennenwein, S.T.B.; Klaui, M. Spin Hall magnetoresistance in the non-collinear ferrimagnet GdIG close to the compensation temperature. J. Phys. Condens. Matter 2018, 30, 035802. [CrossRef]

12. Thi, T.N.; Van, P.C.; Viet, D.D.; Quoc, V.D.; Ahn, H.; Cao, V.A.; Kang, M.G.; Nah, J.; Park, B.G.; Jeong, J.R. Morphology-dependent spin Seebeck effect in yttrium iron garnet thin films prepared by metal-organic decomposition. Ceram. Int. 2021, 47, 16770-16775. [CrossRef]

13. Zhang, D.; Jin, L.; Zhang, H.; Yang, Q.; Rao, Y.; Wen, Q.; Zhou, T.; Liu, C.; Zhong, Z.; Xiao, J.Q. Chemical epitaxial growth of nm-thick yttrium iron garnet films with low Gilbert damping. J. Alloys Compd. 2017, 695, 2301-2305. [CrossRef]

14. Dongquoc, V.; Kuchi, R.; Van, P.C.; Surabhi, S.; Lee, S.W.; Kim, D.; Jeong, J.R. Enhancing magneto-optical and structural properties of Bi-YIG thin film on glass substrate using poly[vinylpyrrolidone](PVP) assisted MOD method. Ceram. Int. 2019, 45, 20758-20761. [CrossRef]

15. Yang, B.; Xia, S.Y.; Zhao, H.; Liu, G.; Du, J.; Shen, K.; Qiu, Z.; Wu, D. Revealing thermally driven distortion of magnon dispersion by spin Seebeck effect in $\mathrm{Gd}_{3} \mathrm{Fe}_{5} \mathrm{O}_{12}$. Phys. Rev. B 2021, 103, 054411. [CrossRef]

16. Liensberger, L.; Kamra, A.; Maier-Flaig, H.; Geprägs, S.; Erb, A.; Goennenwein, S.T.B.; Gross, R.; Belzig, W.; Huebl, H.; Weiler, M. Exchange-enhanced ultrastrong magnon-magnon coupling in a compensated ferrimagnet. Phys. Rev. Lett. 2019, 123, 117204. [CrossRef]

17. Becker, S.; Ren, Z.; Fuhrmann, F.; Ross, A.; Lord, S.; Ding, S.; Wu, R.; Yang, J.; Miao, J.; Kläui, M.; et al. Magnetic Coupling in $\mathrm{Y}_{3} \mathrm{Fe}_{5} \mathrm{O}_{12} / \mathrm{Gd}_{3} \mathrm{Fe}_{5} \mathrm{O}_{12}$ Heterostructures. Phys. Rev. Appl. 2021, 16, 014047. [CrossRef]

18. Isaac, N.; Ruizi, L.; Zheyu, R.; Se kwon, K.; Qiming, S. Survey of temperature dependence of the damping parameter in the ferrimagnet $\mathrm{Gd}_{3} \mathrm{Fe}_{5} \mathrm{O}_{12}$. IEEE Trans. Magn. 2022, 58, 1-13. [CrossRef]

19. Kim, D.H.; Okuno, T.; Kim, S.K.; Oh, S.H.; Nishimura, T.; Hirata, Y.; Futakawa, Y.; Yoshikawa, H.; Tsukamoto, A.; Tserkovnyak, Y.; et al. Low Magnetic Damping of Ferrimagnetic GdFeCo Alloys. Phys. Rev. Lett. 2019, 122, 127203. [CrossRef]

20. Okuno, T.; Kim, S.K.; Moriyama, T.; Kim, D.H.; Mizuno, H.; Ikebuchi, T.; Hirata, Y.; Yoshikawa, H.; Tsukamoto, A.; Kim, K.J.; et al. Temperature dependence of magnetic resonance in ferrimagnetic GdFeCo alloys. Appl. Phys. Express 2019, 12, 093001. [CrossRef]

21. Kim, K.J.; Kim, S.K.; Hirata, Y.; Oh, S.H.; Tono, T.; Kim, D.H.; Okuno, T.; Ham, W.S.; Kim, S.; Go, G.; et al. Fast domain wall motion in the vicinity of the angular momentum compensation temperature of ferrimagnets. Nat. Mater. 2017, 16, 1187-1192. [CrossRef]

22. Jungfleisch, M.B.; Chumak, A.V.; Kehlberger, A.; Lauer, V.; Kim, D.H.; Onbasli, M.C.; Ross, C.A.; Kläui, M.; Hillebrands, B. Thickness and power dependence of the spin-pumping effect in $\mathrm{Y}_{3} \mathrm{Fe}_{5} \mathrm{O}_{12} / \mathrm{Pt}$ heterostructures measured by the inverse spin Hall effect. Phys. Rev. B 2015, 91, 134407. [CrossRef]

23. Jungfleisch, M.B.; Zhang, W.; Jiang, W.; Chang, H.; Sklenar, J.; Wu, S.M.; Pearson, J.E.; Bhattacharya, A.; Ketterson, J.B.; Wu, M.; et al. Spin waves in micro-structured yttrium iron garnet nanometer-thick films. J. Appl. Phys. 2015, 117, 17D128. [CrossRef]

24. Jermain, C.L.; Aradhya, S.V.; Reynolds, N.D.; Buhrman, R.A.; Brangham, J.T.; Page, M.R.; Hammel, P.C.; Yang, F.Y.; Ralph, D.C. Increased low-temperature damping in yttrium iron garnet thin films. Phys. Rev. B 2017, 95, 174411. [CrossRef] 